\title{
Mathematical Structure and Empirical Content
}

\author{
Michael E. Miller
}

\begin{abstract}
Approaches to the interpretation of physical theories provide accounts of how physical meaning accrues to the mathematical structure of a theory. According to many standard approaches to interpretation, meaning relations are captured by maps from the mathematical structure of the theory to statements expressing its empirical content. In this paper I argue that while such accounts adequately address meaning relations when exact models are available or perturbation theory converges, they do not fare as well for models that give rise to divergent perturbative expansions. Since truncations of divergent perturbative expansions often play a critical role in establishing the empirical adequacy of a theory, this is a serious deficiency. I show how to augment state-space semantics, a view developed by Beth and van Fraassen, to capture perturbatively evaluated observables even in cases where perturbation theory is divergent. This new semantics establishes a sense in which the calculations that underwrite the empirical adequacy of a theory are both meaningful and true, but requires departure from the assumption that physical meaning is captured entirely by the exact models of a theory.
\end{abstract}

1. Introduction. Accounts of the interpretation of physical theories have developed in conjunction with, and in several important cases are attendant to, accounts of the structure of scientific theories. In other cases novel commitments about interpretation are tacitly adopted in the work of those interpreting particular physical theories. While there are significant differences between the accounts, many share a common set of commitments. Ruetsche has provided a helpful characterization of what is shared between the standard approaches. She explains that according to them, "... to interpret a theory is to characterize the worlds possible according to it. These worlds are (i) models (in something like the logician's sense) of the theory, and (ii) characterized as physical" (Ruetsche 2011, p. 7). Standard approaches to interpretation consist in an account of how physical meaning accrues to the mathematical structure of the theory.

Even amongst standard approaches, commitments about the second step in Ruetsche's schema are widely variegated. Some accounts explicitly take the connection between mathematical structure and empirical content to be a map of a particular sort, such as an isomorphism, a partial isomorphism, or an embedding. On other accounts the connection is specified less formally and consists of a metaphysical articulation of the structure of the worlds picked out by the theory, with the mathematical structure functioning as a guide. Commitments about the first step of Ruetsche's schema are comparatively well-regimented. To specify the models of the theory, one must

${ }^{\dagger}$ Department of History and Philosophy of Science, University of Pittsburgh

Email: michael.miller@pitt.edu 
stipulate the states, dynamics, and kinematics characteristic of its structure. Ruetsche's caveat that these structures are models of the theory "in something like the logician's sense" is required because physicists are more permissive about structure specification than logicians. ${ }^{1}$ Axiomatizations in mathematical physics typically are given in terms of the mathematical objects most natural for the description of the domain in question, whether they be those of differential geometry, functional analysis, or some other branch of mathematics. $^{2}$ This is what differentiates axiomatically characterized models of mathematical physics from the models of formal logical systems. Standard interpreters all agree about the norms of structure specification in that they all require models to exactly satisfy the dynamical equations of the theory, or the axioms that characterize its content.

The aim of this article is to consider a different approach to structure specification. Perturbation theory characterizes models as small deviations from models whose structure can be characterized exactly. This technique is used widely in physical practice, and sometimes it is resorted to as a matter of calculational convenience. For this reason, it is not typically regarded by philosophers as a method of structure specification, but instead as a useful approximation scheme for extracting numerical predictions from exact models. However, in a number of important cases including quantum field theory and string theory, the best available characterization of the structure of empirically adequate models is provided by perturbation theory. This creates tension with the norms of structure specification accepted by standard interpreters because in these cases no exact model is available. The absence of an exact model results from the fact that resorting to perturbation theory often results in divergence. The approximation does not converge to a new exact model. For this reason it is not clear how to apply standard accounts of interpretation to empirically adequate models of quantum field theory. In face of this problem it is typically assumed, though often only tacitly, that there is some exact divergence-free structure to which we currently do not have access lying behind the success of the theory. On this view, structures satisfying the standard interpreters' norms of structure specification are supposed to underly the empirical success and physical meaningfulness of the theory.

This article pursues a different response to the divergences of perturbation theory. I argue that perturbation theory should not be regarded as an approx-

\footnotetext{
${ }^{1}$ (L. Ruetsche, personal communication, 18 September 2016)

${ }^{2}$ For example, triples $\left\langle M, T_{a b}, G_{a b}\right\rangle$ that exactly satisfy the Einstein field equations are models of general relativity, and the collection of n-point functions for the $\phi_{2}^{4}$ field theory is a model of quantum field theory because they can be shown to exactly satisfy the the Wightman axioms.
} 
imation scheme, but instead that it provides a novel connection between the mathematical structure of a theory and its empirical content. The strategy I pursue to argue for this claim is to fix on one explicitly articulated approach to interpretation, the state-space semantics view developed by Beth and van Fraassen. ${ }^{3}$ I argue that state-space semantics exemplifies Ruetsche's characterization of the standard approach to interpretation. While focusing on one particular approach to interpretation limits the generality of the conclusions established, state-space semantics exhibits a core feature of most standard accounts by taking meaning relations to derive from the existence of maps from the exact structure of the theory to statements expressing its empirical content. I argue that while state-space semantics adequately captures cases in which perturbation is convergent, it fails to adequately capture the empirical success resulting from truncations of a large class of divergent perturbative expansions. ${ }^{4}$ I show that this class cannot be interpreted as an approximation to an exact model. This observation motivates taking seriously the idea that the empirical content of a theory can have limited precision, that is, that empirical content can be inexact. I provide an alternative semantics that does capture the empirical success of truncated divergent expansions by articulating principled limits on their precision. Exact models play a privileged role in attributions of physical meaning to the mathematical structure of theories in standard approaches to interpretation. This has had the adverse effect of preventing physical meaning from accruing to empirically adequate models of quantum field theory. I provide an alternative approach to interpretation which allows for meaningful attributions of physical content to the models of the theory that actually make contact with the world.

The argument proceeds as follows. In the second section I consider an example of divergent perturbation theory in quantum field theory. I then review the necessary elements of the mathematical theory of divergent perturbative expansions. In the third section I argue that state-space semantics adequately captures the case in which perturbation theory yields convergent expansions for observables, but fails to do so for divergent perturbation theory. While truncations of convergent expansions can naturally be interpreted as approximations, the same is not true of divergent expansions. The aim is not to demonstrate a deficiency of state-space semantics in particular. Rather, its role in the argument is simply to provide a concrete target which is explicitly formulated. The assumptions it adopts are also adopted in many other prominent approaches to the attribution of physical meaning to mathematically expressed theories. As such, the criticism I provide of state-space se-

3 (Beth 1960; van Fraassen 1970)

${ }^{4} \mathrm{~A}$ truncation of an infinite expansion is the sum of a finite initial segment of the series. 
mantics in this article applies to many standard accounts. ${ }^{5}$ The third section also illustrates the limitations of state-space semantics using examples from classical mechanics and non-relativistic quantum mechanics. ${ }^{6}$ These are cases in which truncations of divergent perturbative expansions have been an important factor in establishing the empirical adequacy of a theory. Such cases require a semantics different from state-space semantics and I provide such an alternative in Section Four. The final section concludes by emphasizing that dismissing divergent perturbation theory as merely an approximation scheme has the pernicious effect of divorcing physical meaning from empirically adequate models of quantum field theory.

2. The divergence of perturbation theory. Perturbation theory is the predominant method for deriving empirical information from physical theories. $^{7}$ Suppose one wants to evaluate an expression in a theory involving a function, $f(x)$, whose exact structure is not necessarily known. ${ }^{8}$ The function can be perturbatively evaluated by substituting $f(x) \rightarrow \sum_{n=0}^{\infty} c_{n} x^{n}$. For this procedure to be effective, $x$ must be a small parameter describing a deviation from a model that can be characterized exactly. Perturbation theory converges if the sequence of partial sums $S_{N}=\sum_{n=0}^{N} c_{n} x^{n}$ converges to a finite limit, that is, $\lim _{N \rightarrow \infty} S_{N}<\infty$. Of course, if the limit exists it is unique and so when perturbation theory converges it uniquely determines the exact value of the function it is being used to characterize. Perturbation theory is said to diverge if the sequence of partial sums diverges, that is, $\lim _{N \rightarrow \infty} S_{N}=\infty$. In either case, the series can be truncated at some order $N$, and early terms in the series can be summed, $\sum_{n=0}^{N} c_{n} x^{n}$, to give a finite estimate of the value of the function.

While convergent expansions are considered mathematically well-understood, divergent expansions have widely been regarded with suspicion since their discovery. This suspicion is often motivated by appeal to the following remark of Abel: "Divergent series are the invention of the devil, and it is shameful to base on them any demonstration whatsoever ..." (Abel and Holmboe 1839). This section addresses how convergent and divergent perturbation theory differ as attempts to specify the structure of a theory.

\footnotetext{
${ }^{5}$ The details of the argument for that claim will be left to future work.

${ }^{6}$ The large-order divergences I am concerned with have mistakenly been thought to be a special feature of quantum field theory. The argument is actually applicable to a wide variety of physical theories.

${ }^{7}$ Comparatively little philosophical attention has been dedicated to this method. Noteable exceptions include (Batterman 1997; Batterman 2002; Batterman 2007).

${ }^{8}$ The object being perturbatively evaluated need not be a function. Whether it is a function, an eigenvalue, an S-matrix element, an n-point function, or some other structure, the discussion below applies equally well.
} 
Before turning directly to this task, it is instructive to consider the following example. The magnetic moment of the electron, $g$, is a property of electrons when they are exposed to an external magnetic field. Its value according to the Dirac equation, which treats electrons as relativistic particles, is exactly 2. In 1947, experimental evidence revealed that the value of $g$ deviated just slightly from this exact value. ${ }^{9}$ This evidence motivated physicists to search for a theoretical prediction of the anomalous magnetic moment of the electron, $a_{e}=(g-2) / 2$. The value of this observable can be calculated in quantum electrodynamics, a perturbatively characterized model of quantum field theory. Schwinger calculated the first term in the perturbative expansion which generated a value that correctly predicted the value of $a_{e}$ within experimental error. ${ }^{10}$ This successful empirical prediction played a critical role in convincing physicists to pursue relativistic quantum field theory as the framework for describing elementary particle physics. ${ }^{11}$ It is important to note that what provided the theoretical prediction in this case was the truncation of an infinite perturbative expansion at its very first term.

The calculation of terms beyond the first order of perturbation theory becomes increasingly difficult. ${ }^{12}$ The anomalous electron magnetic moment continues to function as a precision test of quantum electrodynamics and the Standard Model of particle physics, and so considerable effort has been dedicated to calculating additional orders. ${ }^{13}$ The difficulty of the calculation is so great that the current state of the art only allows for the determination of five orders. ${ }^{14}$ When these five terms are summed and compared to the experimentally measured value one finds that:

$$
\begin{aligned}
a_{e}(\text { theory }) & =0.001159652180(73)^{15} \\
a_{e} \text { (experiment) } & =0.001159652181(78) \cdot{ }^{16}
\end{aligned}
$$

Agreement between theory and experiment to ten decimal places is an indication that the theory gets something about the world profoundly correct.

\footnotetext{
${ }^{9}$ (Kusch and Foley 1947)

${ }^{10}$ (Schwinger 1948; Schwinger 1949)

${ }^{11}$ The history of the role of this calculation in demonstrating the empirical adequacy of quantum electrodynamics is recounted in detail in (Schweber 1994).

${ }^{12}$ This is the case because the number of Feynman graphs contributing to an individual order grows approximately factorially in the order. The complexity of the integral corresponding to each graph also grows with the order.

13 (Kinoshita 2014)

${ }^{14}$ More specifically, it is fifth-order in the fine structure constant which is proportional to the square of the coupling, so it is tenth-order in the coupling.

${ }^{15}$ (Aoyama, Hayakawa, Kinoshita, and Nio 2012)

${ }^{16}$ (Hanneke, Fogwell, and Gabrielse 2008)
} 
This agreement is often cited as evidence that quantum electrodynamics is the most empirically successful physical theory ever created, and for good reason. Feynman famously explained that the success of this calculation is tantamount to theoretically determining the distance between Los Angeles and New York to the width of a human hair (Feynman 1985, p. 7).

There is, however, a serious problem lurking in the background. There are compelling arguments going back to (Dyson 1952) that suggest that the perturbative calculation diverges if summed to all orders. ${ }^{17}$ This means that as additional orders of perturbation theory are added to the sum, eventually the theoretical prediction will not only deviate from the experimentally determined value, but it will become infinite. The theory, it seems, is not empirically adequate at all: it is infinitely wrong about the value of $a_{e}$. Abel's caution about using such series thus seems to have been warranted. Trusting truncations of divergent series at low orders seems to lead to serious errors. In fact, immediately following his cautionary note, Abel remarks that: “. . That most of these things [truncation of divergent series] are correct, in spite of that, is extraordinarily surprising. I am trying to find a reason for this; it is an exceedingly interesting question" (Abel and Holmboe 1839). The sense in which the truncations work is that the sum of the low orders of divergent perturbation theory often yield values in close agreement with empirically determined values. In the century following Abel's remarks, mathematicians developed a completely rigorous theory of divergent expansions. While this mathematical theory provides many critical pieces of the explanation of their empirical success, below I argue there is an additional philosophical problem which needs to be resolved in order to understand how they hold empirical content.

When divergent perturbation theory is empirically successful, it is usually an indication that the perturbative series in question is asymptotic to some exact structure. ${ }^{18}$ A function $f(x)$ is asymptotic to the series $\sum_{n=0}^{\infty} a_{n} x^{n}$, $f(x) \sim \sum_{n=0}^{\infty} a_{n} x^{n}$, as $x \rightarrow 0$ if and only if for every fixed $N$,

$$
\lim _{x \rightarrow 0} \frac{f(x)-\sum_{n=0}^{N} a_{n} x^{n}}{x^{N}}=0 .
$$

Asymptoticity is a condition that requires that the difference between the exact value of the function and the partial sum of the series that is asymptotic

\footnotetext{
${ }^{17}$ In particular, the arguments suggest that it diverges even after regularization and renormalization techniques are employed to render every individual term of the series finite. This additional complication is discussed in more detail in Section 4.

${ }^{18}$ This explanation of the success of the anomalous electron magnetic moment calculation was offered by Dyson immediately upon arguing for the divergence of the calculation (Dyson 1952).
} 
to it is appropriately small, but not necessarily zero, for every order of perturbation theory. This is a different type of condition than the one required for convergence to the exact value of a function. Convergence requires that in the limit where all orders of perturbation theory are included, the difference between the partial sums of the series and the exact value of the function becomes exactly zero.

The order-by-order representation of the function provided by an asymptotic series exhibits qualitatively different behavior from that provided by a convergent series. When a series is convergent to a function, typically the first few partial sums give a rather poor approximation to the function. As additional orders are included, the approximation eventually become better, and as the series is convergent, in the limit where all of the terms are included the approximation becomes an exact representation of the function. Asymptotic series behave differently. Their first few partial sums typically give very close agreement with the exact value of the function. However, when additional orders are included eventually the partial sums begin to exhibit increasingly poor agreement with the exact value. In the limit where all of the terms are included, since the asymptotic series is typically divergent, it gives a value that is infinitely different from the exact value. This is precisely the behavior of the series representation of the anomalous electron magnetic moment. It is in this sense that the conjecture that the first few partial sums come from an asymptotic series explains the empirical success of the ultimately divergent expansion.

The effectiveness of the truncation of a convergent expansion at approximating a function is accounted for by the fact that the expansion uniquely determines the function when summed to all orders. It is natural to wonder if asymptotic expansions similarly constrain the exact structure lying behind their success. To answer this question, the first relevant observation to make is that each function has a unique asymptotic expansion: if the limit in the definition of asymptoticity exists, it follows immediately that it is unique. However, two different functions can have the same asymptotic expansion. To see why this is the case, consider $f(x)=e^{-1 / x}$ for $x>0$. Then $f(x) \cdot x^{-N} \rightarrow 0$ as $x \rightarrow 0$, so $f(x)$ is asymptotic to the series that has all zero coefficients. It follows that $f(x)$ can be added to another function $g(x)$ with non-trivial asymptotic expansion to generate a new function $h(x)=f(x)+g(x)$, which has the same non-trivial asymptotic expansion as $g(x)$. Repeated application of this argument can, of course, generate an infinite collection of functions which do not agree on their exact value anywhere, but which all share the same asymptotic expansion. Thus, the conjecture that a perturbative expansion is asymptotic to an exact structure does not uniquely specify what 
structure that happens to be.

Fortunately, there is a condition stronger than that the series is asymptotic to the function, but weaker than that the series is convergent, that does determine the function uniquely. This is the strong asymptotic condition. A function $f(z)$, which is analytic in a sector of the complex plane,

$$
S=\left\{z|0<| z|<B ;| \arg z \mid<\frac{1}{2} \pi+\epsilon\right\}
$$

is said to obey the strong asymptotic condition and have $\sum_{n=0}^{\infty} a_{n} z^{n}$ as its strong asymptotic series if there exist $C$ and $\sigma$ such that,

$$
\left|f(z)-\sum_{n=0}^{N} a_{n} z^{n}\right| \leq C \sigma^{N+1}(N+1) !|z|^{N+1},
$$

for all $N$ and $z \in S .^{19}$ Strong asymptoticity, like asymptoticity, is a condition that requires the differences between the exact value of the function and its series representation be appropriately small for every order of perturbation theory. This condition is important because if $\sum_{n=0}^{\infty} a_{n} z^{n}$ is a strong asymptotic series for analytic functions $f(z)$ and $g(z)$ in a sector $S$, it follows that $f(z)=g(z)$ for all $z \in S$. The strong asymptotic series of a function uniquely determines the function, just as a convergent series does. If one knows the strong asymptotic series of a function, then the function can be uniquely reconstructed by Borel summation. Suppose that $\sum_{n=0}^{\infty} a_{n} z^{n}$ is a strong asymptotic series for $f(z)$ in the sector $S$, as defined above. The Borel transform,

$$
g\left(z^{\prime}\right)=\sum_{n=0}^{\infty} \frac{a_{n}}{n !} z^{\prime n},
$$

can be used to produce the unique reconstruction of $f(z)$ from its strong asymptotic series, because for all $z$ such that $|z|<B$ and $|\arg z|<\epsilon$,

$$
f(z)=\int_{0}^{\infty} g\left(z^{\prime} z\right) e^{-z^{\prime}} d z^{\prime}
$$

A series is Borel summable if and only if it is strongly asymptotic to a function. When a series is Borel summable in this manner, the series uniquely determines the function just as in the case of convergent expansions.

These results are of central importance for assessing the extent to which structure specification is possible using perturbation theory. When perturba-

${ }^{19}$ Discussion of the origin of the motivation for this bound can be found in (Reed and Simon 1978). 
tion theory is convergent, the perturbative expansion provides what is necessary for exact structure specification. The discussion of this section shows that when perturbation theory diverges, this question is more subtle. If the series resulting from the perturbative characterization of a model satisfies the strong asymptotic condition, the series suffices to exactly specify the structure of the theory. One is naturally led to wonder whether all instances of empirically successful truncations of divergent perturbation theory, including the calculation of $a_{e}$, can be explained using this fact. If the strong asymptotic condition is not satisfied, the series by itself does not suffice to pin down the exact structure of the theory. If this is the case for the expansion for $a_{e}$, it is not at all clear what the truncated series tells us about the exact model underwriting this empirically successful calculation.

3. State-space semantics. State-space semantics, a view due to Beth and van Fraassen, exemplifies the standard account of how mathematical structure supports the meaning of physical claims. ${ }^{20}$ This section investigates whether or not state-space semantics adequately captures the truth conditions for models that are characterized perturbatively. I show that the answer is negative in the case of models that are not Borel-summable. It should be noted though that this is not intended as a critique of only state-space semantics. My aim is to make plausible that a similar problem faces all approaches to interpretation that rely on a map from an exact mathematical structure to empirical content. The role of state-space semantics is simply to provide a concrete proposal in which exact and perturbative models can be directly compared.

The semantics consists of three ingredients; a state-space, a collection of elementary physical statements, and a satisfaction function. Many physical theories introduce an abstract mathematical state-space, $S$, to represent physical quantities in space and time. Models of the theory are trajectories in the state-space that exactly satisfy the syntactic expression of the dynamical equations of the theory. The elementary physical statements are a collection of sentences, $U(m, r, t)$, expressing the empirical content of the theory. Each $U(m, r, t)$ ascribes a physical magnitude, $m$, a definite value, $r$, at a specific time, $t$. The truth values of the $U(m, r, t)$ depend on the state of the actual physical system being represented. This dependence is captured by a map $h$, the satisfaction function, which connects the mathematical model to the

\footnotetext{
${ }^{20}$ The view is introduced and discussed in (Beth 1960; van Fraassen 1967; van Fraassen 1970; Arntzenius 1991). Debates concerning the semantics of scientific theories in general have largely been supplanted by debates about the interpretation of particular theories, but as I noted, van Fraassen's state-space semantics view exemplifies Ruetsche's characterization of the standard approach to interpretation.
} 
expression of the empirical content of the theory. van Fraassen explains that: "For each elementary statement $U$ there is a region $h(U)$ of the state-space $[S]$ such that $U$ is true if and only if the system's actual state is represented by an element of $h(U)$ " (van Fraassen 1970, p. 328). ${ }^{21}$ The system's actual state is represented by $U$ if measurement of the quantity $m$ at time $t$ would yield precisely the value $r$ ascribed to it by $U$. In other words, if measurement would yield a value, $\bar{r}$, the satisfaction function is the one that assigns "true" to those $U(m ; r ; t)$ with $r=\bar{r}$ and "false" to the others. The region $h(U)$ is the collection of states that yield $r=\bar{r}$. Note that this abstract characterization of the semantics straightforwardly exemplifies both stages of Ruetsche's account of standard approaches to interpretation. Structure specification consists of fixing on the trajectories in state-space that exactly satisfy the relevant dynamical equations of motion, and the kinematic constraints. The characterization of the models as physical occurs through a rule connecting statements expressing the empirical content of the theory to states of the system represented in state-space.

Applying this abstract characterization of the semantics to a particular theory requires choosing the appropriate state-space, elementary physical statements, and satisfaction function for the theory in question. To represent the motions of masses interacting through forces, classical mechanics employs an abstract state-space which encodes the positions, $q_{k}=\left(q_{x}, q_{y}, q_{z}\right)$, and momenta, $p_{k}=\left(p_{x}, p_{y}, p_{z}\right)$, of each mass. The state-space is thus $\mathbb{R}^{6 n}$ for $n$ the number of masses. The dynamics of the theory are defined by a Hamiltonian, $H$, which expresses the nature of the interaction between the masses. Models of the theory are trajectories in state-space that exactly satisfy the canonical equations of motion:

$$
\frac{d q_{k}}{d t}=\frac{\partial H}{\partial p_{k}} \quad \frac{d p_{k}}{d t}=-\frac{\partial H}{\partial q_{k}} .
$$

All of the physical observables described by the theory are functions of the $q_{k}$ and $p_{k}$. The elementary physical statements ascribe values to these observables. For example, one particular $U(m, r, t)$ is the sentence ascribing to a particular physical mass a particular position in space at a particular time. The satisfaction function is the one that assigns "true" to those states in state-space that yield that exact value at the appropriate time, and "false" to the other states.

This semantics adequately captures truth conditions for truncations of convergent perturbation theory. Consider a classical mechanical system of two masses interacting gravitationally. In this case an exact solution to the

\footnotetext{
${ }^{21}$ I have changed van Fraassen's notation for the state-space to avoid confusion below.
} 
dynamical equations of the theory can be found, and state-space semantics can be straightforwardly applied. Moreover, perturbative treatment of such models yields convergent expansions whose limits agree by necessity with the exact solutions to the dynamical equations. This makes it possible to treat truncations of the series representation of the exact solutions as approximations to those solutions. For my purposes, a mathematical object $O_{1}$, can be treated as an approximation of another mathematical object $O_{2}$ if $O_{1}$ is appropriately close to $\mathrm{O}_{2}$ in some sense that is appropriate for the context. It is a relation that obtains between two mathematical objects, independent of their physical interpretation. ${ }^{22}$ When the objects in question are a function and a truncation of a series representation of that function, a measure of the relevant notion of closeness is given by $\left|f(x)-\sum_{n=0}^{N} a_{n} x^{n}\right|$. Truncations of perturbative expansions for solutions of two-body gravitational systems are approximations of the exact solutions that they converge to when summed to all orders. When they are interpreted as approximations, the empirical success of such truncations is accounted for by state-space semantics. The exactly true physical claim with respect to the semantics is the one generated by the mapping from the exact solution. The numerical value provided by the truncation of the perturbative expansion approximates this exact numerical value, and the empirical content of this truncation is underwritten by the exact solution.

More, as usual, is different. When an additional mass is added to the system being represented, an exact solution to the dynamical equations for all time is not currently available. Under certain conditions, the existence of an exact solution can be shown to exist, but its exact form has not been explicitly constructed. ${ }^{23}$ This is an obstacle to the application of state-space

\footnotetext{
${ }^{22}$ There exists a vast literature on approximation and idealization which does not always carefully distinguish between the terms. (McMullin 1985) provides an account of many of the relevant distinctions. The notion of approximation I employ agrees with the one articulated in (Norton 2012). It also agrees with the one articulated by Frigg and Hartmann, who hold up truncations of series expansions as a paradigmatic example of approximation: "One mathematical item is an approximation of another one if it is close to it in some relevant sense. What this item is may vary. Sometimes we want to approximate one curve with another one. This happens when we expand a function into a power series and only keep the first two or three terms. ... The salient point is that the issue of physical interpretation need not arise. Unlike Galilean idealization, which involves a distortion of a real system, approximation is a purely formal matter." (Frigg and Hartmann 2012).

${ }^{23}$ This is an over-simplification. No closed form analytic solution is available, but Sundman was able to construct an exact solution in terms of convergent infinite series. For discussion of Sundman's results see (Barrow-Green 2010; Saari 1990; Siegel 1941). I am grateful to Gordon Belot for bringing this work to my attention. Unfortunately, Sundman's solution requires far too many terms to generate sufficient accuracy to be of any
} 
semantics: in the absence of an explicitly constructed exact solution, it is not possible to explicitly construct a satisfaction function to connect the mathematical structure of the theory with its empirical content. This is not a mere mathematical curiosity, but rather a critical problem of physical practice. Three-body gravitational systems such as the Sun, Earth, and Moon, and the Sun, Saturn, and Jupiter played an important role in investigations of celestial mechanics in the 19th century. In the absence of explicitly constructed exact solutions, the perturbative treatment of the three-body problem took on a role of increased importance. It was the only method available to generate numerical information to compare with the available empirical data. The addition of the third mass can be treated as a perturbation of the exact solution for two gravitating bodies. Perturbative evaluation of the first few partial sums for the trajectories of the planets generated empirical values that matched closely with the available astronomical data. However, it also became clear that when summed to all orders, the expansions diverge. ${ }^{24}$

This case reveals a potentially serious problem for state-space semantics. The perturbative calculation yields infinity for the value of a measurable physical observable and so the semantics regards the theoretical value generated by the perturbative calculation as false. It does not have the resources to assign meaningfulness to the empirically adequate early partial sums. But the problem is actually worse than assigning "false" to the calculations that demonstrated the empirical adequacy of the theory: an argument can be made that it actually treats such calculations as meaningless. Recall that divergent perturbation series do not uniquely correspond to a function. Even when a series can be shown to be asymptotic to a function, there are an infinite collection of other functions to which the series is also asymptotic. This inhibits interpreting the perturbative expansion as an approximation, as the object to which it is supposed to be an approximation is indeterminate. Put simply, the problem for state-space semantics is that it renders the statements that express the empirical adequacy of the theory as at best false, and at worst meaningless. There is, however, an escape option available to the defender of state-space semantics that is very much worth considering. In particular, if the relevant perturbative expansions could be shown to satisfy the strong asymptotic condition and be uniquely associated with a function, the interpretation as an approximation would once again become viable. ${ }^{25}$

use for actual use in comparing with empirical data.

${ }^{24}$ The second volume of Poincaré's The New Methods of Celestial Mechanics is largely a collection of theorems establishing the divergence of all of the different perturbative methods in use at the time (Poincaré 1993). In fact, this analysis led Poincaré to develop the notion of asymptoticity introduced in the previous section.

${ }^{25}$ Unfortunately, the strong asymptotic condition was not developed until long after the 
An example from non-relativistic quantum mechanics serves to illustrate how this escape option for the state-space semanticist might proceed. The state-space of non-relativistic quantum mechanics is a Hilbert space, and the dynamics of the theory is given by the Schrödinger equation,

$$
i \hbar \frac{\partial \Psi}{\partial t}=H \Psi
$$

where $H$ is the Hamiltonian. The physically measurable quantities described by the theory are represented by Hermitian operators on the Hilbert space in which the wavefunction, $\Psi$, is defined. The $U(m, r, t)$ are thus sentences assigning a particular eigenvalue $r$ to a particular Hermitian operator $m$ at particular time. The satisfaction function $h(U)$ assigns "true" to the collection of states yielding the correct eigenvalue for the operator, and "false" to the others. As in the classical case, this semantics can be straightforwardly applied when exact solutions to the Schrödinger equation are available.

The difficulties for state-space semantics arise when exact solutions are not available and appeal must be made to perturbation theory to gain information about systems of interest. An interesting example is provided by the Stark effect which describes the splitting of atomic energy levels in the presence of an external electric field. It is described naturally by the standard formalism for non-relativistic quantum mechanics, with the Hamiltonian $H=-\Delta-$ $Z / r+2 F x_{3}$, where

$$
\Delta=\frac{\partial^{2}}{\partial x_{1}^{2}}+\frac{\partial^{2}}{\partial x_{2}^{2}}+\frac{\partial^{2}}{\partial x_{3}^{2}}, \quad r=\left(x_{1}^{2}+x_{2}^{2}+x_{3}^{2}\right)^{1 / 2},
$$

$Z$ is the atomic number, and $F$ defines the strength of the field along the $x_{3}$ direction. The problem can be treated as a perturbation around the exact solution for the case where the external field is zero. Calculation of the first few orders of perturbation theory yields close agreement with the experimentally observed splittings in atomic spectra. The measurement of this effect played an important role in motivating the transition from the old quantum theory to its modern formulation. ${ }^{26}$ But just as in the previous cases I have discussed in this article, it can be shown that when summed to all orders the perturbative expansions that provide empirical success at low orders ultimately diverge. State-space semantics thus seems to face the same problem in this case as it did in the case of three-body classical gravitating systems.

period during which celestial mechanics was developed, and as a consequence the Borel summability of the relevant expansions remain, to my knowledge, unchecked.

${ }^{26}$ The role of the Stark effect in the history of this transition is recounted in detail in (Duncan and Janssen 2014). 
That is, the statements that express the empirical success of the theory come out as either false or meaningless with respect to the semantics. What differentiates this case from the previous one is that perturbation theory for eigenvalues of the Stark effect Hamiltonian have been rigorously shown to satisfy the strong asymptotic condition. They can be Borel summed and uniquely associated with an exact value. ${ }^{27}$ As noted above, this restores the viability of treating the empirically successful truncations as approximations to the exact eigenvalues. On this view, the existence of the exact eigenvalues underwrites the physical meaningfulness of the perturbative calculation.

The analysis of this section shows that state-space semantics is well-suited for capturing truth conditions for statements expressing the empirical content of a theory in some circumstances. Specifically, it is completely adequate when the structure specification of the theory conforms to the norms of structure specification insisted upon by Ruetsche's standard interpreter. However, when structure is specified perturbatively I have argued that the status of state-space semantics requires careful scrutiny. Analysis of the case of the Stark effect raises the hope that all perturbative expansions that generate empirical success satisfy the strong asymptotic condition. If this were the case, then the success resulting from the truncation of divergent series would always be underwritten by an exact model which the truncation approximates.

Unfortunately, this hope is dashed in the case of empirically adequate models of quantum field theory. ${ }^{28}$ The cause of the failure of Borel summability in quantum field theory is the presence of singularities in the Borel transform due to instantons and renormalons. ${ }^{29}$ These singularities result from Feynman graphs which produce growth in the amplitude proportional to $n$ ! for $n$ the order of perturbation theory. The presence of such singularities on the positive real axis of the Borel transform inhibits the integration required to Borel sum a divergent asymptotic series. ${ }^{30}$ The retreat to treating truncations of perturbative expansions as approximations is thus not available in empirically adequate models of quantum field theory. This means that the calculations that establish the empirical adequacy of quantum electrodynamics, such as the determination of $a_{e}$, cannot be treated as approximations.

\footnotetext{
${ }^{27}$ Borel summability was originally shown in (Graffi and Grecchi 1978). Additional discussion and references can be found in (Simon 1982).

${ }^{28}$ For discussion see (Duncan 2012). He explains that "... the property of Borel summability is an extremely fragile one, and one which we can hardly ever expect to be present in interesting relativistic field theories" (Duncan 2012, p. 403).

${ }^{29}$ Detailed discussion of the significance of these singularities for the problem of structure specification is provided in (Miller 2016b).

${ }^{30}$ Preliminary investigation of simplified models of string theory suggest that the situation is similar in that context (Pasquetti and Schiappa 2010).
} 
Accounting for their success requires departure from standard accounts of how mathematical structure underwrites physical meaning.

4. State-space semantics for divergent perturbation theory. The task of interpreting quantum field theory has been thought to be especially difficult because in its empirically adequate formulation, the norms of structure specification accepted by standard interpreters are not satisfied. Halvorson explains that:

...philosophers of physics have taken their object of study to be theories, where theories correspond to mathematical objects (perhaps sets of models). But it is not so clear where "quantum field theory" can be located in the mathematical universe. In the absence of some sort of mathematically intelligible description of QFT, the philosopher of physics has two options: either find a new way to understand the task of interpretation, or remain silent about the interpretation of quantum field theory. (Halvorson and Muger 2006, pp. 731-732)

In Halvorson's view, the only available mathematically intelligible characterization of the structure of quantum field theory is provided by axiomatization. The particular axiomatization that he prefers enjoys the property that its models satisfy the norms of structure specification accepted by standard interpreters. However, the models that generate empirical success when characterized perturbatively have not been shown to satisfy the axioms. If one wants to interpret these perturbatively characterized empirically adequate models, one must depart from standard approaches to interpretation. A similar sentiment is expressed by Ruetsche:

Given a theory $T, \ldots$ we confront the exemplary interpretive question of how exactly to establish a correspondence between $T$ 's models and worlds possible according to $T$. That is, we confront that question if $T$ is the sort of thing that has models. 'A collection of partially heuristic technical developments' isn't readily attributed a set of models about whose underlying ontology or principles of individuation philosophical questions immediately arise. This isn't to say that 'a collection of partially heuristic technical developments' is unworthy of philosophical attention. It is in itself a philosophically provocative circumstance that such a collection can enjoy stunning empirical success. ${ }^{31}$ (Ruetsche 2011,

\footnotetext{
${ }^{31}$ The characterization of perturbative field theory as "a collection of partially heuristic technical developments" is a reference to a remark of Segal aimed at motivating axiomatic approaches to the theory (Segal 1959, p. 341).
} 
p. 102-103)

The remarks of both Halvorson and Ruetsche amount to insistence that structure specification meets the norms of standard interpreters. ${ }^{32}$ Pace Halvorson and Ruetsche, in my view an adequate approach to interpretation must show how a theory's expression of physical content underwrites its stunning empirical success. The interpretation of physical theories is of philosophical interest because it informs our understanding of the actual world, not possible worlds that differ essentially from our own. That standard approaches to interpretation are unable to accommodate this success is a sign of their inadequacy for establishing how physical meaning attaches to mathematical structure.

There is a response available to defenders of standard interpretation: they can attribute the empirical success of perturbative field theory to the existence of an exact mathematical structure to which we simply do not currently have access. One might reasonably hope to gain access to such a structure in one of the following ways. First, it could turn out that additional work by constructive field theorists will show empirically adequate perturbatively characterized models to satisfy an existing axiomatization of quantum field theory. There exists evidence that they do not, but none of it is definitive and so this remains an open possibility. Alternatively, a new axiomatization of quantum field theory could be developed and empirically adequate models could be shown to exactly satisfy this new axiomatization. ${ }^{33}$ For standard interpreters, showing that quantum field theory underwrites the greatest empirical successes ever achieved with a physical theory is predicated on the hope that such a structure will be discovered.

The argument of the previous section lays the foundation for a departure from standard interpretation according to which physical meaning can be associated with empirically successful perturbatively characterized models, without appealing to hoped for exact mathematical structures. Pursuing this alternative approach requires first recognizing that perturbatively characterized models are entirely mathematically intelligible, and are not merely "a collection of partially heuristic technical developments." The formalism for perturbative evaluation is subject to a number of well-known mathematical problems that have led to pessimism about its mathematical meaningfulness. These problems including infrared and ultraviolet divergences, which render individual orders of perturbation theory infinite. However, it is essential

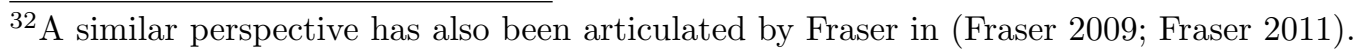

${ }^{33}$ Realizing either of these possibilities would likely amount to solving the Clay Mathematics Institute Millenium Problem on quantum Yang-Mills theory with a mass gap. The problem is stated in (Jaffe and Witten 2000), and additional discussion can be found in (Douglas 2004).
} 
to note that for renormalizable theories like quantum electrodynamics, perturbative renormalization theory provides a conceptually clear and physically motivated procedure for rendering every individual order of perturbation theory finite. ${ }^{34}$ Furthermore, the output of this procedure is a mathematically well-defined formal power series which one can attempt to sum. ${ }^{35}$ The convergence properties of the series when summed to all orders are thus the only legitimate challenge to the structure specification of perturbatively characterized models. ${ }^{36}$

Accounting for how mathematical structure holds empirical content in face of large-order divergences that are not Borel summable requires departure from the norms of structure specification accepted by standard interpreters. In my view, divergent perturbation theory provides a novel connection between mathematical structure and physical meaning that should be incorporated into the semantics of a theory. ${ }^{37}$ State-space semantics can be modified in a way that captures this novel connection. The new semantics retains the core aspects of state-space semantics. The role of the state-space and the elementary physical statements remain unchanged. Modifications are only required for the definition of the satisfaction function.

The first observation at the heart of the modification is that van Fraassen's articulation of state-space semantics relies on an unrealistic characterization of measurements. ${ }^{38}$ Actual measurements never determine the exact value of a quantity. Agreement between theory and experiment instead takes the form of comparisons of a theoretically determined value, $r$, and an experimentally

\footnotetext{
${ }^{34}$ For discussion of infrared divergences and how they are related to the problem of structure specification see (Earman and Fraser 2006) and (Miller 2016a). Ultraviolet divergences are addressed in (Wallace 2006), (Wallace 2011), (Williams 2016), and (Fraser 2016). The approach of Wallace, Williams, and Fraser resolves the problem of ultraviolet divergences by treating empirically adequate field theories as effective theories whose empirical content is confined to a limited scale. I develop an approach to resolving the problem for structure specification raised by ultraviolet divergences in a manner which is compatible with an effective field theory interpretation, but which does not require one, in (Miller 2016c).

${ }^{35}$ If the reader doubts this claim I encourage them to consult (Wightman 1986).

${ }^{36}$ Note that I am not claiming that all calculations found in the physics literature on quantum field theory are rigorous. The claim is that there is a subset of such calculations that suffice to run my argument that can be made rigorous.

${ }^{37} \mathrm{~A}$ number of other authors have recently called into question standard assumptions about how physical content accrues to mathematical structure (Curiel 2011; Ruetsche 2011; Wilson 2006). The departures from standard interpretation that they argue for are not directly connected to the one I advocate in this article, however.

${ }^{38}$ I believe van Fraassen would agree. He explains that "The exact relation between $U(m ; r ; t)$ and the outcome of an actual experiment is the subject of an auxiliary theory of measurement, of which the notion of 'correspondence rule' gives only the shallowest characterization (van Fraassen 1970, p. 329)."
} 
determined value, $\bar{r}$, with some associated measurement error $\epsilon_{m}$. Theoretically determined values are empirically adequate not just when $r=\bar{r}$, but when $r \in\left(\bar{r}-\epsilon_{m}, \bar{r}+\epsilon_{m}\right)$. The first required modification to the semantics is to redefine the satisfaction function so that it returns "true" not just when $r=\bar{r}$, but also when $r \in\left(\bar{r}-\epsilon_{m}, \bar{r}+\epsilon_{m}\right)$.

The core issue for state-space semantics raised by the divergence of perturbation theory concerns the role of the theoretically determined value in the semantics. The nature of the modification to the measured value suggests that there is some freedom in the theoretical values that are compatible with measurement results. This freedom can be exploited to provide a new semantics that adequately captures the empirical success of truncations of perturbative expansions that are convergent, strongly asymptotic, and even those that are not Borel summable. The details are different for each case, but the central idea is to provide a well-defined bound on the error introduced by truncating the expansion, $\epsilon_{t}$. If that bound can be shown to be compatible with the freedom resulting from the presence of the measurement error, $\epsilon_{m}$, the truncation error can be seamlessly incorporated into the semantics.

For convergent and strongly asymptotic series, finding such a principled bound is typically straightforward. In the case of convergent Taylor series for example, the relevant bounds can be provided by results related to Taylor's theorem. Consider the Taylor series of $f(x)$ about the point $a, \sum_{n=0}^{\infty} c_{n}(x-a)^{n}$ for $c_{n}=f^{(n)} / n$ !. If $\left|f^{(n+1)}(x)\right| \leq M$ for all $x \in(a-r, a+r)$ with some $r>0$, then the error from truncation at the $N$ th term, $f(x)-\sum_{n=0}^{N} c_{n}(x-a)^{n} \equiv$ $R_{N}(x)$, is bounded by

$$
\left|R_{N}(x)\right| \leq M \frac{|x-a|^{N+1}}{(N+1) !} .
$$

The truncation error, $\epsilon_{t}$, can be taken to be,

$$
\epsilon_{t}=M \frac{|x-a|^{N+1}}{(N+1) !} .
$$

Similarly, it has already been noted in the Section Two that for a series that is strongly asymptotic to a function in the sector $S$, there exist $C$ and $\sigma$ such that,

$$
\left|f(z)-\sum_{n=0}^{N} a_{n} z^{n}\right| \leq C \sigma^{N+1}(N+1) !|z|^{N+1},
$$

for all $N$ and $z \in S$. In this case we can take,

$$
\epsilon_{t}=C \sigma^{N+1}(N+1) !|z|^{N+1}
$$


There are also principled methods for assigning error bounds to truncations of divergent asymptotic expansions that are not Borel summable. Suppose $f(x) \sim \sum_{n=0}^{\infty} a_{n} x^{n}$. The optimal truncation rule dictates that the series should be truncated at the smallest term of the series, $N_{\min }$, so that the value of the truncation is $\sum_{n=0}^{N_{\min }} a_{n} x^{n}$. This rule is justified by the fact that the minimal error is typically achieved with this truncation. Moreover, the error is typically bounded by the magnitude of the value of the least term, $\left|f(x)-\sum_{n=0}^{N_{\min }} a_{n} x^{n}\right| \leq\left|a_{N_{\min }} x^{N_{\min }}\right|$. The caveat "typically" is important, and both properties must be rigorously confirmed for each individual case. But in those situations where they can be confirmed we can take,

$$
\epsilon_{t}=a_{N_{\min }} x^{N_{\min }} .
$$

These rigorously established bounds on the truncation error provide the critical ingredient to complete the modified semantics. The satisfaction function must be redefined so that in addition to accounting for the measurement error, it is also ensured that the truncation error is not greater than the freedom allowed in the theoretical value by the measurement error. This is the case when $\left(r-\epsilon_{t}, r+\epsilon_{t}\right) \subset\left(\bar{r}-\epsilon_{m}, \bar{r}+\epsilon_{m}\right)$. The satisfaction function thus needs to be redefined so that the $U(m, r, t)$ are true when $\left(r-\epsilon_{t}, r+\epsilon_{t}\right) \subset$ $\left(\bar{r}-\epsilon_{m}, \bar{r}+\epsilon_{m}\right)$ and false otherwise. A new feature of the view is that the empirical content of a theory can come with precisely defined, but limited precision.

A number of remarks are in order. In the previous section I argued that truncations of convergent and strongly asymptotic expansions can be interpreted as approximations. When this approach is taken, all of the physical meaning derives from the exact model, and the truncation simply approximates the exact value. But, the proposal of this section makes it clear that it is not necessary to view such truncations as approximations. On the alternative view developed here, convergent and strongly asymptotic expansions are not interpreted as approximations. They are to be interpreted in just the same way as truncations of series that are not Borel summable for which the interpretation as an approximation is not available. It is these limited precision comparisons to experiments that convinced physicists of the truth of the theory in each of the cases introduced above. For this reason I believe this is the best way to capture the semantics of divergent perturbation theory, even in cases where Borel summability is retroactively established. Another important thing to note is that nothing about this proposed modification to state-space semantics involves a lack of mathematical rigor. When the bounds used for $\epsilon_{t}$ are established by the means discussed above, their existence is proved by the standards of rigor accepted by mathematicians. 
The most important advantage of this proposal is that it allows us to treat the most empirically successful theories as providing meaningful claims about the world. Moreover, it does so by making minimal, and, in my view, natural modifications to an approach to interpretation that exemplifies the core commitments of standard interpretation. The modifications are minimal as all that is involved is a redefinition of the satisfaction function. They are natural in the sense that the modification that is made is directly motivated by the nature of the empirical support for the theory being interpreted. Rather than adhering to philosophical commitments about how theories ought to hold physical meaning in their mathematical expression, the account captures how they actually do hold empirical content in physical practice.

There are two counterintuitive consequences of the modified state-space semantics that I have developed in this section which must be weighed against the advantages just articulated. The first is explained in the following remark of Magnen and Rivasseau:

Constructive field theory builds functions whose Taylor expansion is perturbative field theory. Any formal power series being asymptotic to infinitely many smooth functions, perturbative field theory alone does not give any well defined mathematical recipe to compute to arbitrary accuracy any physical number, so in a deep sense it is no theory at all. (Magnen and Rivasseau 2008, p. 403)

Perturbative field theory is not a theory in the sense that it cannot be given a state-space semantics, or any interpretation that requires that there be physical facts of the matter about the exact value of physical observables. On my view, the empirical content of the theory simply has a limited, but rigorously established, precision. This is counterintuitive, but I think it is worth asking why we default to the assumption that there is a physical fact of the matter about the trillionth decimal place of physical observables, let alone the $10^{1000}$ th decimal place. I am not aware of any physical observable for which there are empirical grounds for commitment to this level of precision. Defenders of standard interpretation owe an explanation for their tacit commitment to this view, and it should be one based on something other than philosophical preconceptions about the structure of scientific theories.

The second counterintuitive consequence is that the truth values vary with experimental precision. The modified satisfaction function requires that $\left(r-\epsilon_{t}, r+\epsilon_{t}\right) \subset\left(\bar{r}-\epsilon_{m}, \bar{r}+\epsilon_{m}\right)$, and over the course of time, $\epsilon_{m}$ can be made smaller with improvements in experimental techniques. This points to another important difference between convergent and divergent expansions. For convergent expansions, $\epsilon_{t}$ can always be made arbitrarily small by 
summing additional orders of perturbation theory. This is not the case for divergent asymptotic expansions, whether they are strongly asymptotic to a function or not. Asymptoticity and strong asymptoticity only assure that the error induced by truncation at a particular order is small, and there is some order for which this error is minimized. If the measurement error is eventually reduced beyond this minimum truncation error, on my view the theory no longer expresses the empirical content of the theory sufficiently precisely to be confirmed by experiments. I am not aware of any case in actual physical practice where this possibility has been realized, but such a case would certainly warrant careful analysis. While both of these counterintuitive consequences merit further discussion, I believe the advantages of the modified semantics developed here outweigh any negative considerations they bring to bear on my view.

5. Conclusion. Consider once more Abel's question: why do truncations of perturbative expansions generate empirically adequate values? Defenders of standard interpretation naturally resort to treating truncations as approximations to exact models. However, this route is not available in cases where perturbation theory is not Borel summable. To account for the empirical success of quantum electrodynamics, for example, they have no choice but to wait for some new exact structure to underwrite the success of calculations of observables like $a_{e}$. If the norms of structure specification accepted by standard interpreters are to be met, this is the only option.

I have offered an alternative approach to answering Abel's question. On my view, perturbation theory presents a genuinely novel connection between mathematical structure and physical meaning. By incorporating this connection directly into the semantics for physical theories, we can meaningfully account for the empirical successes of quantum field theory. Rather than hoped-for structure, I have advocated that we look to the methodologies used in physical practice. In one sense this is conservative. The modification to state-space semantics that I advocate is minimal in the sense that it preserves most of the features of the view as expressed by van Fraassen. It is natural in the sense that the modifications to state-space semantics are motivated by the nature of the empirical support for the theory in question. But in another sense it is radical. It requires that we accept that expressions of the empirical content of physical theories can have in principle limits on their precision, and that the truth values of statements expressing that empirical content might vary with the precision of measurements. In my view, the benefits of having a firm sense of how theories as we actually have them make contact with the world far outweigh the luxury of rigidly maintaining philosophical commitments about the structure of theories. 
6. Acknowledgements. I am thankful to Dave Baker, Gordon Belot, Erik Curiel, James Fraser, Sebastian Lutz, John Norton, Laura Ruetsche, Porter Williams, and audiences at the University of Michigan and Ludwig-MaximiliansUniversität for helpful discussion of the subject of this article. I am especially grateful to Bob Batterman who, to borrow a phrase from Tai Tsun $\mathrm{Wu}$, is responsible for transmitting the large-order disease to me. 


\section{References}

Abel, N. and B. Holmboe (1839). Oeuvres complètes de N.H. Abel, mathématicien, avec des notes et développements: rédigées par ordre du Roi, Volume 2. C. Gröndahl.

Aoyama, T., M. Hayakawa, T. Kinoshita, and M. Nio (2012). Quantum electrodynamics calculation of lepton anomalous magnetic moments: Numerical approach to the perturbation theory of QED. Progress of Theoretical and Experimental Physics 2012, 01A107.

Arntzenius, F. (1991). State-Spaces and Meaning Relations Among Predicates. Topoi 10(1), 35-42.

Barrow-Green, J. (2010). The dramatic episode of sundman. Historia Mathematica 37(2), $164-203$.

Batterman, R. W. (1997). Into a Mist: Asymptotic theories on a caustic. Studies in History and Philosophy of Science Part B: Studies in History and Philosophy of Modern Physics 28(3), 395-413.

Batterman, R. W. (2002). The Devil in the Details: Asymptotic Reasoning in Explanation, Reduction, and Emergence. Oxford University Press.

Batterman, R. W. (2007). On the Specialness of Special Functions (The Nonrandom Effusions of the Divine Mathematician). The British Journal for the Philosophy of Science 58(2), 263-286.

Beth, E. W. (1960). Semantics of Physical Theories. Synthese 12(2-3), $172-175$.

Curiel, E. (2011). On the propriety of physical theories as a basis for their semantics. http://philsci-archive.pitt.edu/8702/.

Douglas, M. (2004). Report on the status of the Yang-Mills millenium prize problem. http://www.claymath.org/sites/default/files/ym2.pdf.

Duncan, A. (2012). The Conceptual Framework of Quantum Field Theory. Oxford University Press.

Duncan, A. and M. Janssen (2014). The trouble with orbits: The Stark effect in the old and the new quantum theory. Studies in History and Philosophy of Science Part B: Studies in History and Philosophy of Modern Physics 48, 68-83.

Dyson, F. J. (1952). Divergence of Perturbation Theory in Quantum Electrodynamics. Physical Review 85(4), 631-632.

Earman, J. and D. Fraser (2006). Haag's Theorem and its Implications for the Foundations of Quantum Field Theory. Erkenntnis 64 (3), 305-344. 
Feynman, R. (1985). QED: The Strange Theory of Light and Matter. Penguin Books.

Fraser, D. (2009). Quantum Field Theory : Underdetermination, Inconsistency, and Idealization. Philosophy of Science 76(4), 536-567.

Fraser, D. (2011). How to take particle physics seriously: A further defence of axiomatic quantum field theory. Studies in History and Philosophy of Science Part B: Studies in History and Philosophy of Modern Physics 42(2), 126-135.

Fraser, J. D. (2016). What is Quantum Field Theory?: Idealisation, Explanation, and Realism in High Energy Physics. Ph. D. thesis, University of Leeds.

Frigg, R. and S. Hartmann (2012). Models in science. In E. N. Zalta (Ed.), The Stanford Encyclopedia of Philosophy (Fall 2012 ed.).

Graffi, S. and V. Grecchi (1978). Resonances in Stark effect and perturbation theory. Communications in Mathematical Physics 62(1), 83-96.

Halvorson, H. and M. Muger (2006). Algebraic quantum field theory. In J. Butterfield and J. Earman (Eds.), Handbook of the Philosophy of Science, pp. 731-922.

Hanneke, D., S. Fogwell, and G. Gabrielse (2008). New measurement of the electron magnetic moment and the fine structure constant. Physical Review Letters 100, 120801.

Jaffe, A. and E. Witten (2000). Quantum Yang-Mills theory. http://www.claymath.org/prizeproblems/yang_mills.pdf.

Kinoshita, T. (2014). Tenth-order QED contribution to the electron g-2 and high precision test of quantum electrodynamics. International Journal of Modern Physics A29, 1430003.

Kusch, P. and H. M. Foley (1947). Precision measurement of the ratio of the atomic ' $g$ values' in the ${ }^{2} p_{\frac{3}{2}}$ and ${ }^{2} p_{\frac{1}{2}}$ states of gallium. Physical Review 72, 1256-1257.

Magnen, J. and V. Rivasseau (2008). Constructive $\phi^{4}$ Field Theory without Tears. Annales Henri Poincaré 9, 403-424.

McMullin, E. (1985). Galilean idealization. Studies in History and Philosophy of Science Part A 16(3), 247-273.

Miller, M. E. (2016a). Haag's theorem, apparent inconsistency, and the empirical adequacy of quantum field theory. The British Journal for the Philosophy of Science (forthcoming). 
Miller, M. E. (2016b). On the common structure of perturbative and axiomatic field theory in Borel summable models. (In Preparation).

Miller, M. E. (2016c). Why are there ultraviolet divergences at all? (In Preparation).

Norton, J. D. (2012). Approximation and idealization: Why the difference matters. Philosophy of Science 79(2), 207-232.

Pasquetti, S. and R. Schiappa (2010). Borel and Stokes nonperturbative phenomena in topological string theory and $\mathrm{c}=1$ matrix models. $A n$ nales Henri Poincaré 11(3), 351-431.

Poincaré, H. (1993). New Methods of Celestial Mechanics: Approximations by series. History of modern physics and astronomy.

Reed, M. and B. Simon (1978). Methods of Modern Mathematical Physics Volume 4: Analysis of Operators. Academic Press.

Ruetsche, L. (2011). Interpreting Quantum Theories. Oxford University Press.

Saari, D. G. (1990, February). A visit to the newtonian n-body problem via elementary complex variables. Am. Math. Monthly 97(2), 105-119.

Schweber, S. S. (1994). QED and the men who made it: Dyson, Feynman, Schwinger, and Tomonaga. Princeton University Press.

Schwinger, J. (1948). On quantum-electrodynamics and the magnetic moment of the electron. Physical Review 73, 416-417.

Schwinger, J. (1949). On the classical radiation of accelerated electrons. Physical Review 75, 1912-1925.

Segal, I. E. (1959). The Mathematical Meaning of Operationalism in Quantum Mechanics. In L. Henkin, P. Suppes, and A. Tarski (Eds.), The Axiomatic Method With Special Reference to Geometry and Physics, pp. 341-352. Amsterdam: North Holland Publishing Company.

Siegel, C. L. (1941). On the modern development of celestial mechanics. The American Mathematical Monthly 48(7), 430-435.

Simon, B. (1982). Large orders and summability of eigenvalue perturbation theory: A mathematical overview. International Journal of Quantum Chemistry 21(1), 3-25.

van Fraassen, B. C. (1967). Meaning Relations Among Predicates. Nô̂s 1(2), 161-179.

van Fraassen, B. C. (1970). On the Extension of Beth's Semantics of Physical Theories. Philosophy of Science 37(3), 325-339. 
Wallace, D. (2006). In defence of naiveté: The conceptual status of Lagrangian quantum field theory. Synthese 151(1), 33-80.

Wallace, D. (2011). Taking particle physics seriously: A critique of the algebraic approach to quantum field theory. Studies in History and Philosophy of Science Part B: Studies In History and Philosophy of Modern Physics 42(2), 116-125.

Wightman, A. (1986). Some lessons of renormalization theory. In Copenhagen1985 Proceedings, The lessons of quantum theory, pp. 201-226.

Williams, P. (2016). Scientific realism made effective. (In Preparation).

Wilson, M. (2006). Wandering significance: An essay on conceptual behavior. Oxford University Press. 